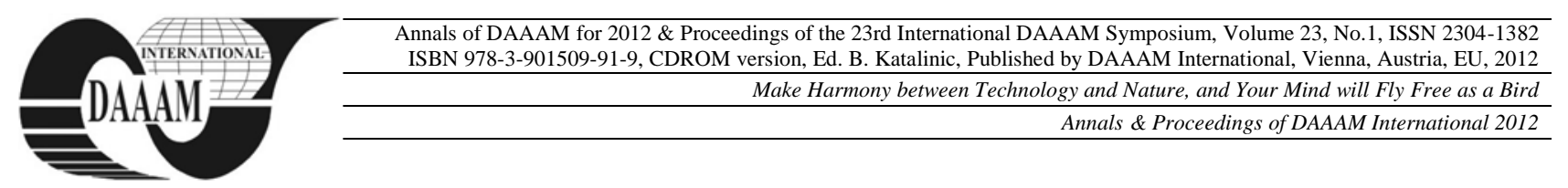

\title{
THE SIMULATION OF MAGNETIC FORCE THAT ACTS ON THE MICRO-ROBOT
}

\author{
SURIANSKY, J[ozef] \& PETRAS, R[udolf]
}

\begin{abstract}
The article deals with the simulation of magnetic force that acts on the micro-robot. The source of magnetic force is the electric coil. As a simulation tool, we used professional software, which is designed for simulation of electromagnetic field. The simulation shall verify the effects of coil force for the move of a micro-robot.

Keywords: micro-robot, simulation, magnetic force, magnetic field
\end{abstract}

\section{INTRODUCTION}

The magnetic field and its ability to operate on wires or charges are currently used in devices designated for many job sectors. In our paper, we are interested in the simulation of magnetic forces for the control of microrobot by the resolution in microns.

Micro-robots may be used for manufacturing, analysis of chemical and biological agents, microorganisms, human cells, micro-particles, etc... In these analyses, operations shall take place so that such measurements of toxicity and sampling will be carried out directly on this place or in the laboratory. [5] In fact, people do not have access to such places because these are extremely dangerous and could be that careless and "rough" handling is causing enormous damage range. In this case, it requires the use of micro-devices (microrobots), which may help us to obtain samples. [1]

With regards to micro-robots and micromanipulators, a research is currently being done mainly in areas of micromachining, surface-micromachining and micromanipulation. [4] Common methods of the application contain the production of sensors, optical micro-assemblies (micro-mirrors assembly in optical switches), digital displays, wireless communication modules and other applications where it is necessary build up micro-components into the desired configuration. [7]

Fig. 1 shows the coils assembly that create controlled magnetic fields. The coils assembly consists of four coils that are located around the workspace that manipulates with magnetic micro-robot. The magnetic force is simulated with connected coil $\mathrm{C}$, which flow a direct current. The micro-robot was continually being moved from the head of coil and there were obtained values of magnetic force. These values were evaluated graphically (Fig. 4).

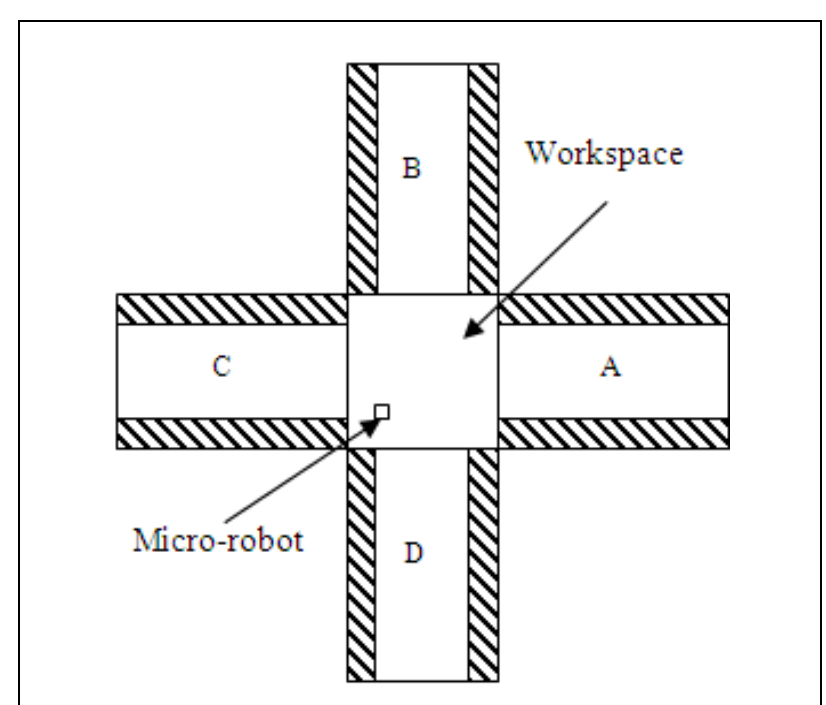

Fig. 1. Block diagram of coils assembly for positioning micro-robot. A, B, C, D - coils

\section{MAGNETIC FORCE BETWEEN MAGNETS}

Repulsive forces are between the permanent magnets. Attractive force causes that the electrons with the electric charges move around the core and the magnetisation of elementary particles of the substance. Both can be described by small current loops called magnetic dipole. The initial force creates between two magnets in the interaction between dipoles. If we know all the magnetic dipoles, which consist of magnets, then the force of two magnets, we can determine the sum of all interactions of dipoles, the first and the second magnet.

\subsection{Magnetic force calculation}

Calculation of attractive and repulsive forces between two magnets is generally a complex process because it depends on the shape, magnetization, orientation and distance of the magnets. The Gilbert's model is based on the knowledge distribution of magnetic charge in a magnetic field. This model is applicable only to simple cases. Equation (1) shows that we can calculate the magnetic force between two dipoles. [3]

$$
F=\frac{\mu q_{m 1} q_{m 2}}{4 \pi r^{2}}
$$

\section{$F-$ force $[\mathrm{N}]$}

$\mu$ - permeability of the intervening medium $[\mathrm{H} / \mathrm{m}]$ $q_{m 1}, q_{m 2}-$ the magnitudes of magnetic poles [A.m] $r$ - the separation $[\mathrm{m}]$ 


\subsection{Force between two magnetized areas}

The equation (2) can be valid only in those cases when the effect of dispersion is a minimum and the volume of air gap is much smaller than the magnetized material.

$$
F=\frac{\mu_{0} H^{2} A}{2}=\frac{B^{2} A}{2 \mu_{0}}
$$

$A$ - the area of each surface $\left[\mathrm{m}^{2}\right]$

$H$ - the magnetic field intensity $[\mathrm{A} / \mathrm{m}]$

$\mu_{0}$ - the permeability of space $\left[4 \pi \cdot 10^{-7} \mathrm{H} / \mathrm{m}\right]$

$B$ - the magnetic field induction [T]

\subsection{Force between bar magnets}

The magnetic force between two bar magnets can be calculated using (3), but the following condition must be met:

- bar magnets shall be identical.

$$
F=\left[\frac{B_{0}^{2} A^{2}\left(L^{2}+R^{2}\right)}{\pi \mu_{0} L^{2}}\right]\left[\frac{1}{x^{2}}+\frac{1}{(x+2 L)^{2}}-\frac{2}{(x+L)^{2}}\right]
$$

$A-$ the area of each surface [m2]

$B_{0}$ - the magnetic field induction very close to each pole [T]

$L$ - the length of each magnet [m]

$R$ - the radius of each magnet [m]

$x$ - the separation between the two magnets [m]

$\mu_{0}$ - the permeability of space $[4 \pi .10-7 \mathrm{H} / \mathrm{m}]$

\section{MICRO-ROBOT}

The micro-robot was in the cube shape with $1 \mathrm{~mm}$ edge. It was derived from neodymium-iron-boron $(\mathrm{NdFeB})$, a hard magnetic material, with the magnetic remanence $1.5 \mathrm{~T}$ and permeability equal one. Fig. 2 shows a simple micro-robot, which was designed to simulate the magnetic force.

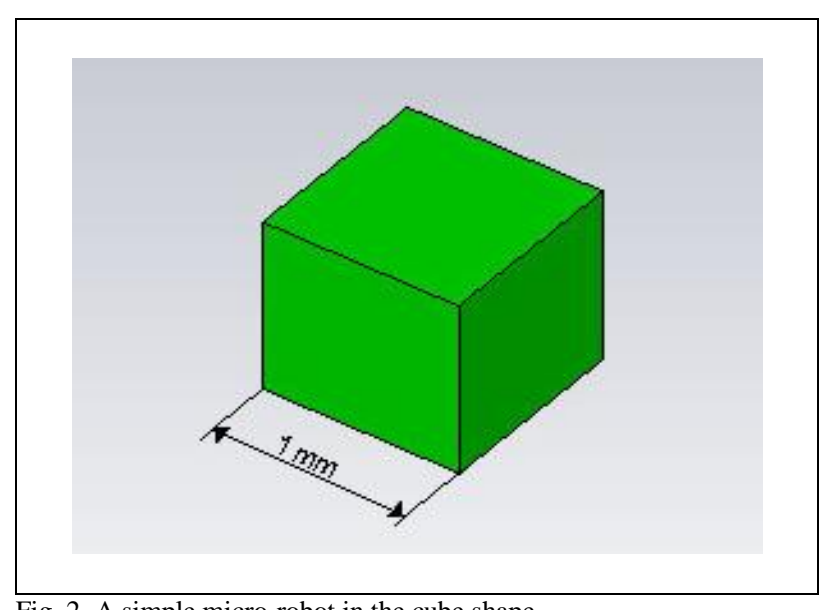

Fig. 2. A simple micro-robot in the cube shape

\section{MAGNETIC FORCE SIMULATION}

The simulation of magnetic force was necessary to verify designed parameters of the coils. Parameters were designed in order to achieve that the coil would be able to position micro-robots at each position in the workspace. Therefore, the power effects of the coil were simulated on a micro-robot. The parameters of coil were designed as follows:

- cross sectional area of core $(\mathrm{A})-78.5 \mathrm{~mm}^{2}$,

- length of the coil (l) $-31 \mathrm{~mm}$,

- inductance $(\mathrm{L})-18.5 \mathrm{mH}$,

- number of turns $(\mathrm{N})-1600$.

The magnetic force was calculated in the specific simulation program CST EM STUDIO that focused on the simulation of the electromagnetic field. The program also supported the calculation of the magnetic force on objects in a magnetic field. In our case, it was a microrobot of permanent magnet.

The simulated environment is shown in Fig. 3. The first step was the placement of micro-robot to the front of the coil $C$. Then, the micro-robot was moved in the perpendicular direction to the front of the coil. Moving dimension was $1 \mathrm{~mm}$. For each displacement, the magnetic force that acted on the micro-robot was acquired. Measured results are shown graphically in Fig. 4. The line shows the minimum size of force necessary to move the micro-robot. The curve shows the size of forces acting on micro-robot depending on its distance from the head coil. The micro- robot movement will be guaranteed when magnetic force, which is showed as curve, is greater than the minimum force required to move.

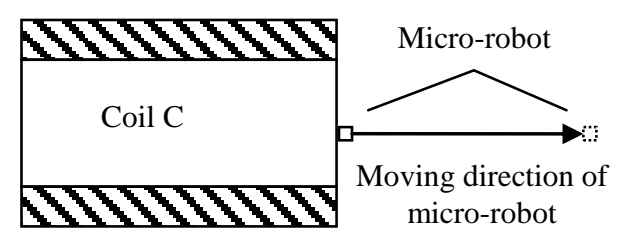

Fig. 3. The simulation model of magnetic force

\section{THE FORCE CALCULATION NECESSARY TO MOVE THE MICRO-ROBOT}

The micro-robot was moved in the workspace that was made of clear plastic. The micro-robot contained compounds of neodymium-iron-boron. Therefore, we performed the calculation of the frictional forces among these materials. Several measurements were done while there were obtained lengths $h$ and $d$ as shown in the Fig. 5. These values were set to (3) and the measurement results were averaged. The result was the coefficient of static friction is equal to 0,37 .

$$
\tan \alpha=\frac{h}{d}
$$

$\alpha=\mu_{s}-$ static friction

$h$ - height of an inclined plane

$d$ - length of horizontal plane 




Fig. 4. The dependence of magnetic force at a distance

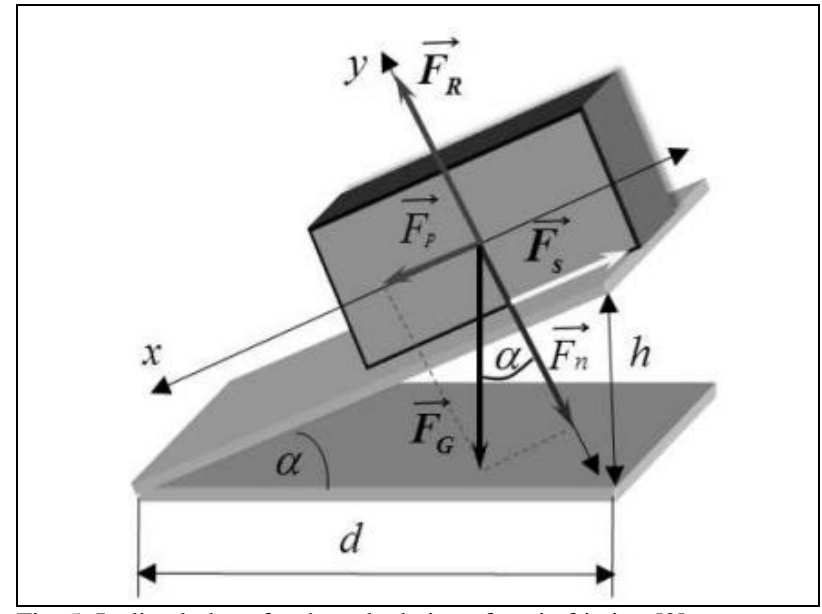

Fig. 5. Inclined plane for the calculation of static friction [2]

The minimum force was calculated from the coefficient of static friction that was necessary for the movement of the micro-robot. Its weight was calculated from the density of rare earth neodymium-iron-boron (composition of micro-robot) and the micro-robot volume. The gravitational force $F_{G}$, which acts perpendicularly down, was obtained from the calculated weight and the gravitational acceleration. The coefficient of static friction $\mu_{\mathrm{s}}$ and gravity force $\mathrm{F}_{\mathrm{G}}$ were multiplied. The result from the formula was the force $F_{\min }$. This force is the minimum force required to move the micro-robot and it is equal to $2,77 \times 10^{-2} \mathrm{mN}$ and show in Fig. 4 .

\section{ADVANTAGES AND BENEFITS}

The benefit of process simulation was the acquisition of necessary data for effective design of the micromanipulator. We can optimize the design of the power system for the current control of the coil. If the power system will be designed, we can modify the control system of the micromanipulator.

The micro-robot uses contactless control using magnetic fields. The working environment of microrobots can be the air or liquid but it shall be specified by the following requirements.

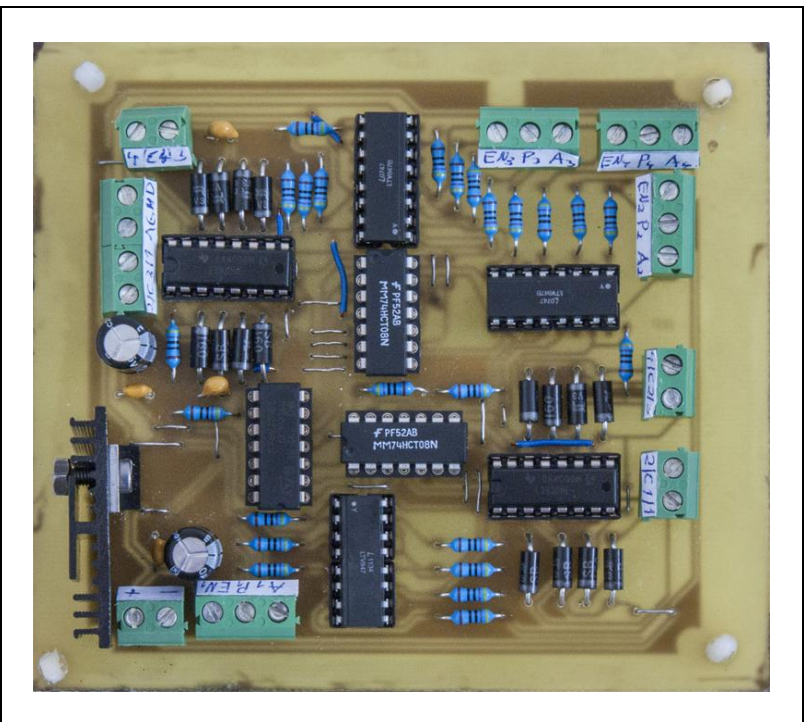

Fig. 6. The experimental model of power system

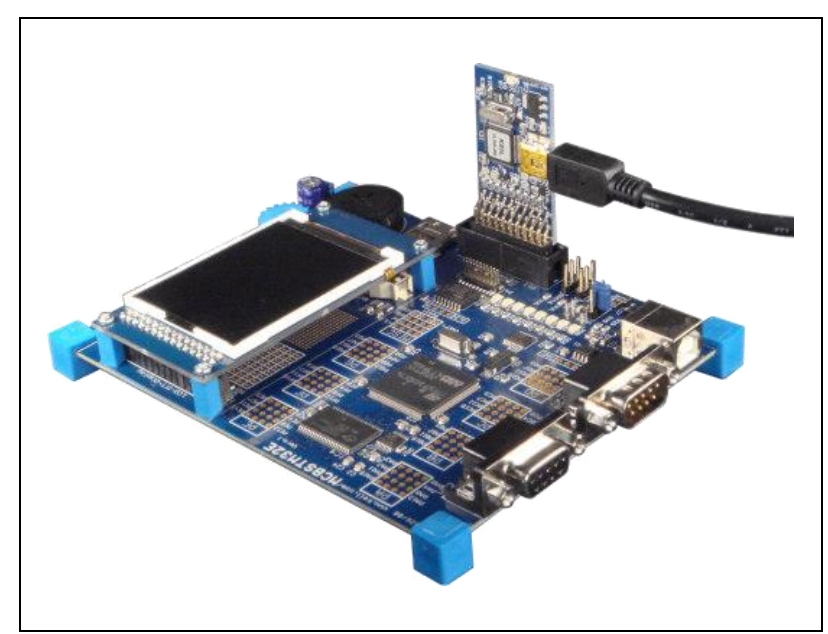

Fig. 7. The control system

The fluid cannot be of high viscosity and cannot be of high friction between the surface and the robot.

The advantage is the use of magnetic material for manufacturing of micro-robots because it does not 
require any other power on its positioning. They are made from available permanent magnets since their production is very cheap and they can be produced in large quantities. Magnets are strongly resistant from weather and other influences regarding their size.

In practice, applications of micro-robots are planned in environments which are inaccessible and dangerous for people [6] such as follows:

- micromachining, surface-micromachining or microassembly,

- dangerous biological and chemical waste in the processing of containers,

- $\quad$ analysis of toxic substances in pipelines

- technical conditions of equipment,

- dangerous physiological processes in plant and animal organisms, and others.

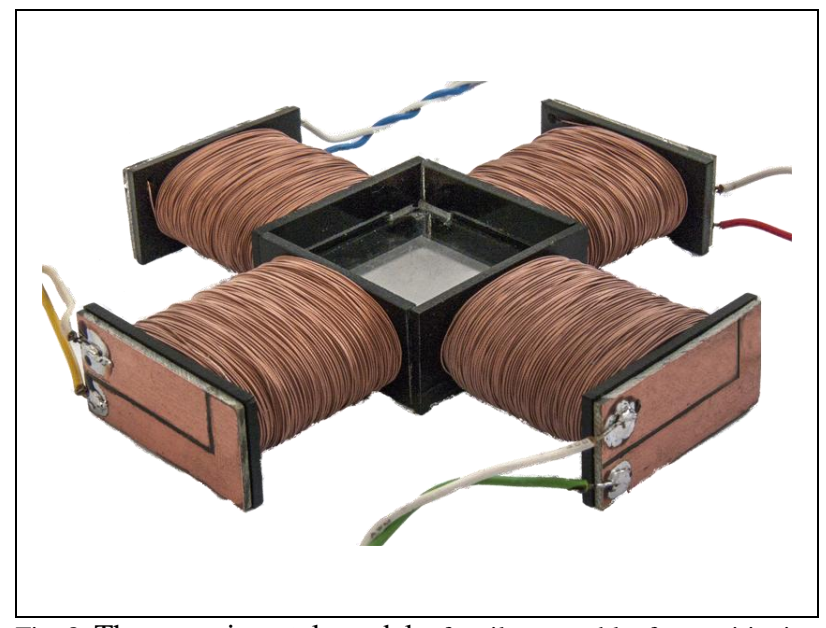

Fig. 8. The experimental model of coils assembly for positioning micro-robot.

\section{CONCLUSION}

The research in manufacture, in natural sciences and the practice in many field is now often required in the micromachining, the analysis of chemical and biological substance, microorganisms, human cells, micro-particles, etc. These analyses are performed by micro-robots. They can be used in the area that is dangerous to humans. The movement of these micro-robots is controlled by the programming of certain tasks or directly managed by no contact way.

The article deals with the simulation of magnetic force that acts on the micro-robot. This simulation was necessary to verify the force effects that create selfdesigned coils. The problem was solved by using the simulator CST EM STUDIO. This program was used to simulate coils and their magnetic field which acted on the neodymium-iron-boron magnet (micro-robot). It was observed that simulated values of forces acting on microrobot are bigger than the minimum force required for its movement.

This knowledge indicates that the micro-robot will be able of a controlled movement in the workspace. The magnetic force coil was designed with sufficient power reserves because the micro-robot is not always rotated on the coil with a different pole. The simulation of multirotation micro-robot has not been performed so far.

The work has been resolved, some sub-tasks as design of power system and design and implementation of the experimental model of coils assembly. In the near future, the design of motion sensors for getting move of micro-robot is planned. The use of these sensors shall ensure a controlled movement of a micro-robot.

\section{REFERENCES}

[1] Shen, Y., Xi, N., Lai, K., Li, W.: A novel PVDF microforce / force rate sensor for practical applications in micromanipulation, Available from: <www.emerald insight.com/researchregister>, 2004, 274str

[2] *** (2012) Základy fyziky, Basics of physics. Available from: <http://hockicko.uniza.sk/Books/ZakladyFyziky_KudelcikHocki cko.pdf>, Accessed: january 2012

[3] Thidé, B:. 2009. Elekromagnetic field theory. Uppsala, Sweden, 262str. ISBN 978-0-486-4773-2

[4] Bogue, R:. 2010. Microrobots and nanorobots: a review of recent developments. Okehampton, UK: Industrial Robot, 2010, ISSN 0143-991X

[5] Bogue, R: \& Partners. 2008. The development of medical microrobots: a review of progress. Okehampton, UK: Industrial Robot, 2008, ISSN 0143-991X

[6] *** (2011) Autonomni mikrorobot, Autonomous micro-robot. Avaible from: <http://www.techblog.cz/nano/autonomnimikrorobot.html>, Accessed:July 2011

[7] *** (2012) Reconfigurable Magnetic Micro-Modules [online]. Avaible from: <http://nanolab.me.cmu.edu/projects/Magneti cMicroRobot/>, web preview January 2012 